Escuela de Ciencias Sociales y Humanidades, UNED, Costa Rica https://revistas.uned.ac.cr/index.php/espiga ISSN: 1409-4002 • e-ISSN: 2215-454X

\title{
Antropoceno, ¿Última unidad geocronológica o llamamiento a la nueva cultura ambiental que necesita la humanidad?
}

\section{Anthropocene: Last geochronological unit or call for the new environmental culture that humanity needs? \\ L'Anthropocène, dernière unité géochronologique ou appel à la nouvelle culture de l'environnement dont I’humanité a besoin?}

Wilfredo Ricardo Mesa-Ortega *

https://orcid.org/0000-0002-8704-6743

Recibido: 26 de octubre de 2020 • Aceptado: 11 de febrero de 2021

* Doctor en Ciencias Pedagógicas por la Universidad de Matanzas, Cuba. Catedrático de Geografía en la Facultad de Educación de la Universidad de Matanzas. Profesor de Geología General y de Educación Ambiental para el Desarrollo Sostenible en la carrera de Geografía. Autor de numerosos artículos sobre educación y cultura ambiental. Es miembro del Grupo de Medio Ambiente en la Universidad de Matanzas. Correo: wilfredo.ricardo59@gmail.com 


\section{Resumen}

Se asiste al debate sobre el Antropoceno como nueva unidad geocronológica, signada por la aceleración de los impactos de la actividad humana sobre el planeta, desde una mirada que promueva la reflexión y participación en las acciones para enfrentar los ingentes riesgos y desafíos ambientales, generadores de gran incertidumbre sobre el futuro de la humanidad y su casa común: la Tierra. Más que mostrar acuerdo o no con este nuevo intervalo geocronológico, se pretende avivar la indagación personal sobre los impactos ambientales del homo sapiens, para percatarse de que los estilos de desarrollo dominantes, los progresos tecnológicos, el crecimiento demográfico y la capacidad alcanzada para impactar sobre los nexos ambientales y ecosistémicos globales, lo han convertido en una fuerza capaz de poner en crisis el equilibrio del mundo. Ante tan compleja situación global, junto con muchas otras soluciones políticas, tecnológicas, económicas y socioculturales, se insiste en la importancia de educar una nueva cultura ambiental y se propone un procedimiento viable para la autovaloración de dicha cultura, concebido como un acto de reflexión crítica y autoconocimiento, proyectado hacia el mejoramiento de la persona y su relación con el medio ambiente en el que vive.

Palabras clave: Autovaloración, crisis civilizatoria, cultura ambiental, escala geocronológica global.

\section{Abstract}

The debate on the Anthropocene as a new geochronological unit is giving rise, marked by the acceleration of the impacts of human activity on the planet, from a perspective that promotes reflection and participation in actions to deal with the enormous risks and environmental challenges, generators of great uncertainty about the future of humanity and its common home: the Earth. Rather than showing agreement or not with this new geochronological interval, it is intended to stoke personal inquiry into the environmental impacts of homo sapiens, to realize that the dominant development styles, technological progress, population growth and the capacity achieved to impact on global environmental and ecosystem ties, have made it a force capable of putting the balance of the world in crisis. Faced with such a complex global situation, along with many other political, technological, economic and socio-cultural solutions, the importance of educating a new environmental culture is insisted on and a viable procedure is proposed for the self-assessment of said culture, conceived as an act of critical reflection and selfknowledge, projected towards the improvement of the person and his/her relationship with the environment in which he/she lives.

Keywords: Civilizational crisis, environmental culture, global geochronological scale, self-assessment.

\section{Résumé}

Nous assistons au débat sur l'Anthropocène comme une nouvelle unité géochronologique marquée par l'accélération des impacts de l'activité humaine sur la planète, dans une perspective que vise à favoriser la réflexion et la participation dans les actions afin de faire face aux grands risques et défis environnementaux, lesquels causent une grande incertitude sur le futur de I'humanité et leur maison: la Terre. Nous ne sommes pas intéressés à discuter si l'on est d'accord ou non avec ce nouvel intervalle géochronologique, notre but est d'éveiller la recherche personnelle sur les impacts environnementaux de l'Homo sapiens afin de prendre conscience que les styles de développement dominantes, les progrès technologiques, la croissance démographique et la 
capacité atteinte pour impacter les liens environnementaux et écosystémiques mondiaux ont transformé l'être humain en une force capable de mettre en danger l'équilibre du monde. Étant donné la situation globale si complexe, des solutions politiques, technologiques, économiques et socioculturelles ont été proposées. Auprès de ces solutions, nous insistons sur l'importance d'éduquer sur une nouvelle culture environnementale et nous proposons un processus possible pour l'autoévaluation de cette culture qui a été conçue comme un acte de réflexion critique et d'autoconnaissance pour l'amélioration de la personne et sa relation avec l'environnement dans laquelle elle vit.

Mots-clés: autoévaluation, crise civilisatrice, culture de l'environnement, échelle géochronologique globale. 


\section{Introducción}

Los intentos de establecer y dar nombre al más reciente intervalo del tiempo geológico, cuya singularidad está marcada por los enormes impactos de la actividad humana sobre el medio ambiente, no son totalmente novísimos. Las experiencias del autor, adquiridas por más de 30 años explicando a educadores de Geografía acerca de los contenidos de Geología General y de Educación Ambiental para el Desarrollo Sostenible, lo estimularon a implicarse en el furor del debate científico actual en torno al Antropoceno y a cuestionarse si constituye la última unidad geocronológica o un llamamiento a la nueva cultura ambiental que necesita la humanidad.

El objetivo que persiguen estas líneas es divulgar la crisis civilizatoria contemporánea, insuficientemente visualizada por muchas personas, a pesar de que pone en riesgo su existencia futura, llámese en el Antropoceno o con otro término, y destacar la importancia de educar una nueva cultura ambiental en todos los niveles; en cuyo contexto de saberes, humanismo práctico y capacidades de gestión, se ayude a solucionar favorablemente la gran paradoja de la humanidad: mientras más crecen los indicadores económicos de producción y consumo, bajo el impulso del desarrollo científico y tecnológico, más se ahondan los nefastos impactos sobre el medio ambiente ${ }^{1}$.

Lo que se presenta no es una sistematización de ideas sobre el Antropoceno y los problemas que lo definen, aunque necesariamente se realizó una revisión bibliográfica para intervenir en el debate y tomar posiciones científicas, éticas y prácticas, acordes con lo más avanzado en el consenso internacional sobre cómo detener y revertir el signo de la crisis medioambiental en que la humanidad se adentra peligrosamente hacia el futuro, sin haber logrado todavía la integración y la justicia ambiental ${ }^{2}$ que hace falta para tomar decisiones acertadas en este sentido y accionar unidos.

Se aspira a que sirva para contrarrestar el discurso catastrofista en torno al futuro de la Tierra y el ser humano, así como para comprender la urgencia de encontrar soluciones a estos problemas del Antropoceno, trabajando en los diferentes niveles de acción y en el lugar que corresponde a cada uno, siempre en la estrecha colaboración de todos los países, culturas y personas, tal como lo soñó José Martíi: «Con todos y para el bien de todos».

\footnotetext{
1 Jeffer Chaparro e Ignacio Meneses, «El antropoceno: aportes para la comprensión del cambio global», Ar@cne. Revista electrónica de recursos en internet sobre Geografía y Ciencias Sociales, n. ${ }^{\circ} 203$ (2015): 4. 2 Liz-Rejane Issberner y Philippe Léna, «Antropoceno: la problemática vital de un debate científico», Correo de la Unesco, 2018-2, acceso: 20 de setiembre de 2021, https://es.unesco.org/courier/2018-2/antropocenoproblematica-vital-debate-cientifico

3 José Martí, Obras Completas: Discurso en el Liceo Cubano, Tampa. Tomo 4 (La Habana: Editorial de Ciencias Sociales, 1991), 279./art06.pdf
} 


\section{Desarrollo}

En 1881, Antonio Stoppani (considerado el padre de la geología italiana por su notable labor científica, didáctica y de divulgación en este campo de saberes), propuso una nueva era: antropozoica o el neozoico, pero en esa fecha su propuesta no tuvo amplia acogida en la comunidad científica, dado que en ese momento histórico la contradicción entre la actividad humana y el equilibrio de la naturaleza no era tan crítica como en la actualidad.

En la década de 1980, el biólogo estadounidense Eugene F. Stoemer utilizó ell término Antropoceno para caracterizar las graves afectaciones ecológicas y de extinción de especies, producidas por causas antropogénicas. En 1992, Andrew Revkin introdujo el vocablo Antroceno para referirse a una posible nueva era geológica marcada decisivamente por la actividad humana en la Tierra. Por su parte, Michael Samways insistió en una idea similar en 1999, aunque propuso que la nueva etapa fuese llamada el Homogenoceno, en el cual la biodiversidad disminuye y los ecosistemas están cambiando por la acción humana.

En su sentido geocronológico, el concepto Antropoceno fue acogido, empleado y muy debatido por una parte de la comunidad científica, según la definición planteada entre los años 2000 y 2002, por el meteorólogo holandés y premio Nobel de Química (1995) Paul Crutzen, quien ubicó su comienzo al final del siglo $\mathrm{XVIII}$, momento en que ya se denotaban con claridad los ingentes cambios que el humano ha generado sobre la faz de la Tierra. Su propuesta se basó en resultados de estudios del aire atrapado en masas de hielo de los polos, los cuales mostraron una elevada concentración de metano y dióxido de carbono antropogénicos, capaz de trasformar las condiciones atmosféricas naturales ${ }^{4}$.

Cuando Crutzen planteó la necesidad de definir el Antropoceno y su comienzo a finales del siglo XVIII, lo que hizo «fue lanzar una pequeña granada de mano hacia el mundo de la escala temporal geológica» ${ }^{5}$, e inmediatamente se crearon grupos de discusión sobre el tema en prestigiosas universidades y sociedades científicas. En 2009 se creó el Grupo de Trabajo sobre el Antropoceno de la Comisión Internacional de Estratigrafía y dicho concepto entró en el lenguaje científico de múltiples eventos y publicaciones, así como de ciencias sociales y humanas, que hasta entonces no se habían interesado en el dominio de los conocimientos geocronológicos y de la historia natural de la Tierra.

Al calor de la polémica entre geólogos, arqueólogos, geógrafos, biólogos, filósofos, historiadores, medioambientalistas, tecnólogos, políticos, religiosos y humanistas en general, han surgido nuevos términos alusivos al Antropoceno, tales como Capitaloceno ${ }^{6}$ y Occidentaloceno, que no han tenido suficiente

\footnotetext{
4 Issberner y Léna, «Antropoceno: la problemática...»

5 Laura Plitt, «¿Qué es el Antropoceno, la "Edad de los humanos" y que expertos aseguran hemos entrado?», BBC Mundo, 30 de agosto de 2016, acceso: 20 de setiembre de 2021, https://www.bbc.com/mundo/noticias37220892

6 «Léxico del Antropoceno», Correo de la Unesco, 2018-2, acceso: 20 de setiembre de 2021, https:// es.unesco.org/courier/2018-2/lexico-del-antropoceno
} 
aceptación entre naturalistas, por su matiz sociopolítico más que geológico, ni entre científicos del mundo capitalista occidental que pretenden evadir la responsabilidad histórica de los principales daños a la naturaleza y al humano mismo, en el lapso geológico que se pretende delimitar.

La revisión de diversos artículos sobre la llegada al Antropoceno -producto de que hay quienes consideran que la humanidad se ha convertido en una fuerza geológica ${ }^{7}$ decisora de los destinos del medio ambiente y la vida terrestremuestra que no todos los autores coinciden en el tipo de unidad geocronológica a que correspondería: unos lo consideran nueva era, otros un período, otros una época, otros una edad y hay quienes proponen que englobe y haga innecesario el Holoceno: la más reciente época del período Cuaternario, reconocida por la comunidad científica, desde el Congreso Geológico Internacional de 1885.

Las imprecisiones terminológicas antes apuntadas oscurecen el debate y denotan descuidos en el uso del lenguaje científico inherente al tema tratado y el poco dominio de los conocimientos básicos de geocronología, particularmente de la escala geocronológica global, conocida popularmente como la tabla del tiempo geológico. Ante la situación precisada conviene preguntarse: ¿A qué tipo de unidad geocronológica y geoestratigráfica de la escala temporal geológica correspondería el Antropoceno?

La geología histórica es la ciencia dedicada a estudiar las transformaciones que ha sufrido la Tierra desde su formación, hace 4650 millones de años, hasta el presente. Todo ha cambiado a lo largo de millones de años: la distribución de continentes y océanos, la formación y destrucción de rocas y relieves, las condiciones climáticas o la posición de los polos geográficos y magnéticos terrestres, así como las formas de vida, incluido el ser humano ${ }^{8}$.

La historia geológica de la Tierra corrobora que el mundo material (y con él la subjetividad humana), está en constante movimiento, en el espacio y a través del tiempo, lo cual se expresa en la evolución continua, la circulación permanente de las sustancias y la energía, el carácter rítmico de estos cambios y la estructura sistémica de la naturaleza, en la que todos sus componentes están interrelacionados complejamente, de forma tal que los cambios en uno de ellos desencadenan cambios en los demás9.

La escala del tiempo geológico es una herramienta elaborada gracias a los estudios de muchos geólogos, paleontólogos y otros naturalistas durante siglos, para representar los principales acontecimientos de la historia de la Tierra, ordenados cronológicamente. No ha incluido en sus contenidos los eventos de la evolución histórica de la humanidad (del homo sapiens), iniciada en el Holoceno, hace alrededor de 11700 años, antes del 2000 y hasta el presente, de los cuales se ocupan, hasta el momento, las ciencias históricas y humanas.

\footnotetext{
7 Shiraz Sidhva, «La humanidad es una fuerza geológica», Correo de la Unesco, 2018-2, acceso: 20 de setiembre de 2021, https://es.unesco.org/courier/2018-2/humanidad-es-fuerza-geologica

8 Wilfredo Mesa, «Geología Elemental». Material de consulta de curso. Licenciatura en Educación BiologíaGeografía y Biología-Química, Universidad de Matanzas. En soporte digital, 2017.

9 Ibíd.
} 
La tabla geológica está integrada por unidades geocronológicas que dividen al tiempo en: eones, eras, períodos, épocas, edades y crones, ordenadas en disposición jerárquica, de igual manera que un extenso libro se divide en tomos, capítulos, epígrafes, acápites y párrafos. Dichas unidades tienen como soporte material a sus equivalentes unidades cronoestratigráficas: eonotemas, eratemas, sistemas, series, pisos y cronozonas; definidas como los paquetes de rocas, estratificadas 0 no, que conservan características bioestratigráficas y litoestratigráficas distintivas, porque se formaron en determinado intervalo de tiempo geológico, bajo condiciones ambientales específicas.

Recapituladas estas nociones, es lógico que hoy retornen las ideas de Antonio Stoppani, referidas al comienzo de una nueva era, aunque se esté iniciando aquí y ahora, por lo que es el presente y no el pasado o la historia de la Tierra; de ahí lo difícil que resulta insertarla en la escala geológica global, con tan solo unos miles de años e insuficientes registros bioestratigráficos y litoestratigráficos que la distingan de la actual era Cenozoica, que se inició hace 66 millones de años y se extiende hasta la actualidad.

Por otra parte, el Antropoceno pudiera ser definido como la más reciente edad del Holoceno, de la cual se registran ya algunos tecnofósiles ${ }^{10}$ y estratos de plastiglomerados; sin embargo, esta posibilidad es casi desechable, ya que, en pleno apogeo del debate científico sobre si debe o no considerarse como un nuevo intervalo de tiempo geológico, en julio de 2018, la Unión Internacional de Ciencias Geológicas ratificó, a propuesta de la Comisión Internacional de Estratigrafía, la definición formal de tres edades holocénicas: Groenlandiense, Norgripiense y Megalayense.

También pudiera situarse el Antropoceno como el período de la era Cenozoica que sobreviene al Cuaternario, para cuyas divisiones en épocas, edades y crones se debiera tomar, como principal indicador, la historia del impacto humano sobre el medio ambiente, además del comportamiento natural de las geosferas terrestres en su compleja interconexión; lo cual significaría una valiosa integración entre las ciencias naturales, sociales y humanas, muy útil para cerrar el paso a los profetas del fin del mundo y enfrentar los enormes desafíos que la humanidad deberá resolver en el futuro antropocénico.

Otro aspecto muy debatido tiene que ver con el comienzo del Antropoceno, ya que se proponen diversas fechas: el inició de la agricultura hace 10000 años atrás, al final del siglo XVIII, después de la Segunda Guerra Mundial o a partir de 1970 con «la gran aceleración» ${ }^{11}$. Todas las posiciones argumentadas generan confusión entre tiempo histórico-geológico y tiempo histórico-humano, ya que los eventos empleados como marcas geológicas son histórico-sociales y no histórico-geológicos.

Además, la brevedad temporal de esta nueva unidad (10 000, 300, 70 o 50 años), cuestiona su necesidad, puesto que el Holoceno fue definido como: «una

\footnotetext{
10 «Léxico del Antropoceno...»
}

11 Ibíd. 
época del Cuaternario iniciada 11000 atrás, más cálida y húmeda, cuando los seres humanos colonizaron nuevos territorios»12. Entonces, cabe preguntarse si es apropiado usar el mismo argumento del impacto de la actividad humana para definir una época posterior. ¿Se estará sobredimensionando el papel de la actividad humana como fuerza geológica en esta etapa de la historia de la Tierra, que también es su presente?

Sin el ánimo de apaciguar el actual debate, ¿Saben quiénes intervienen en la polémica sobre el Antropoceno? $\mathrm{O}$ ¿Cómo se procede para definir formalmente las nuevas unidades en la tabla del tiempo geológico? Esta responsabilidad científica corresponde, desde 1974, a la Comisión Internacional de Estratigrafía, cuyos cambios o novedades que propone, luego de intensos análisis y deliberaciones en subcomisiones multidisciplinarias muy especializadas, deben ratificarse en los congresos mundiales de la Unión Internacional de Ciencias Geológicas.

Para que los especialistas definan nuevas unidades geocronológicas en la tabla del tiempo geológico, es necesario comprobar la existencia de una ruptura universal entre las capas sedimentarias de la nueva unidad y la anterior ${ }^{13}$. Esta ruptura debe reflejar cambios litoestratigráficos (tipos genéticos de rocas, sus texturas y composición química, facies sedimentarias) y bioestratigráficos (restos fósiles y compuestos orgánicos específicos), que evidencien grandes cambios en la dinámica endógena y exógena de los territorios, en las condiciones climáticas, en la vida y los ecosistemas, es decir, las nuevas unidades están basadas en acontecimientos que marcan hitos en el tiempo geológico y permanecen reflejados en las rocas formadas entonces.

Aunque crece el consenso sobre el hecho de que varios parámetros del sistema terrestre han empezado a evolucionar fuera del espectro de variabilidad natural del Holoceno, el concepto de Antropoceno aún no ha sido aprobado por la Unión Internacional de Ciencias Geológicas y la comunidad de geólogos muestra bastante escepticismo hacia su aprobación. No hay dudas de la magnitud alcanzada por la actividad humana sobre la naturaleza, fundamentalmente en los dos últimos siglos, pero no constan suficientes evidencias estratigráficas de los impactos humanos, como tampoco han tenido una distribución espacial y temporal relativamente uniforme a escala global; por tal motivo, el concepto en controversia se mantiene en el terreno de la hipótesis o de la metáfora.

Puede que todavía no se llegue a reconocer una nueva etapa geológica denominada Antropoceno; no obstante, el autor de estas líneas considera que lo más productivo y atrayente de este exaltado debate mundial es haber obligado a buena parte de la comunidad científica, y a la humanidad en general, a reflexionar sobre el lugar que ocupa el ser humano y sus formaciones socioeconómicas, en la historia y evolución del planeta Tierra; percatándose de que los progresos tecnológicos, el crecimiento demográfico y su capacidad para

12 Plitt, «¿Qué es el Antropoceno...»

13 Ibíd. 
extenderse por todas las geosferas, llegando a configurarse el ilusorio pensamiento de que lo domina y utiliza a su antojo, lo han convertido en una enorme fuerza transformadora que ha puesto en grave crisis el equilibrio del mundo.

No cabe duda, salvo en mentes perversas, prepotentes e irresponsables, que la humanidad, en su relación con la naturaleza, vive una crisis actual de carácter cultural, civilizatoria ${ }^{14}$; resultante de la interacción sustentada en una plataforma cultural donde se articulan actividades socioeconómicas, medios de producción y herramientas de trabajo, formas de organización social y construcciones simbólicas (las diversas formas de conciencia social), que hasta el presente han sido de corto plazo y dirigidas a explotar a la naturaleza y los seres humanos, sin atender a su recuperación, desestimando sus propiedades, necesidades y leyes de funcionamiento.

Increíblemente, las alertas de varios científicos preocupados por dicha crisis no fueron tomadas en cuenta cuando aún no era tan grave y hubiese sido más fácil enfrentarla. Uno de ellos fue Engels, fundador, junto a Marx, de la filosofía marxista. En el siglo XIX, aseveró15:

Todo nos recuerda a cada paso que el hombre no domina, ni mucho menos, la naturaleza a la manera como un conquistador domina a un pueblo extranjero, es decir, como alguien que es ajeno a la naturaleza, sino que formamos parte de ella (...), que nos hallamos en medio de ella y que todo nuestro dominio sobre la naturaleza y la ventaja que en esto llevamos a las demás criaturas consiste en la posibilidad de llegar a conocer sus leyes y de saber aplicarlas acertadamente.

No solo desde el ámbito de las ciencias quedaron mensajes precisos sobre la importancia de amar y respetar a la naturaleza, el cubano José Martí16, hombre universal preocupado por todo lo que pudiera mejorar al mundo, escribió poéticamente, también en el siglo XIX:

Y el único camino abierto a la prosperidad constante y fácil es el de conocer, cultivar y aprovechar los elementos inagotables e infatigables de la naturaleza. La naturaleza no tiene celos, como los hombres. No tiene odios ni miedo, como los hombres. No cierra el paso a nadie, porque no teme a nadie. Los hombres siempre necesitarán de los productos de la naturaleza.

En varios de los estudios consultados se plantea que, hasta el presente, el humano se ha creído -o se ha sentido- como el amo de la naturaleza ${ }^{17}$; sin embargo, abundan desde el comienzo mismo de la crisis autores como los antes

\footnotetext{
14 José Mateo, «Medio Ambiente y Desarrollo» (La Habana: Universidad de La Habana. Facultad de Geografía, 2002). En soporte digital.

15 Federico Engels, Dialéctica de la naturaleza. El papel del trabajo en el proceso de transformación del mono en hombre (La Habana: Editora Política, 1979), 151-152.

16 José Martí, Obras Completas: Maestros ambulantes. Tomo 8 (La Habana: Editorial de Ciencias Sociales, 1991), 289.

17 Chaparro y Meneses, «El Antropoceno: aportes...»
} 
citados, quienes demuestran fehacientemente lo errático de dicha concepción. Luego entonces ¿Se ha llegado a esta crisis antropocénica, por falta de conocimientos, por ignorancia del homo sapiens o por una ineficiente gobernabilidad de las sociedades humanas sometidas al capital, en vez de a la razón científica o a los saberes de las culturas humanas ancestrales, en las que se rendía culto a la naturaleza?

La historia pone en evidencia que los conocimientos acumulados por la humanidad sobre las propiedades, necesidades y leyes de funcionamiento de la naturaleza y de la sociedad han sido desestimados de forma deliberada, mayormente después de la Primera Revolución Industrial, ocurrida entre la segunda mitad del siglo XVIII y la primera del XIX, con la expansión del capitalismo, cuyas ganancias proceden de explotar la naturaleza y a los seres humanos, sin prestar atención a su recuperación y satisfacción de necesidades y derechos para la continuidad de su existencia y desarrollo.

Es innegable que algunos teóricos alineados al capitalismo contemporáneo intentan alejar el debate antropocénico de la discusión política ${ }^{18}$, alegando que el impacto de los regímenes socioeconómicos sobre el medio ambiente no es asunto de las ciencias naturales y que dicha crisis es un problema de la especie humana en general, al crear la tecnosfera: nueva capa artificial de la Tierra que crece constantemente por la acción exclusiva del ser humano, compuesta por los objetos que ha creado (desde ciudades, aeropuertos, fábricas, carreteras y monumentos, hasta cosas más pequeñas como ropas, automóviles, computadoras y teléfonos celulares) para satisfacer sus necesidades de consumo mediante la transformación de recursos naturales. Se estima que alcanza un peso total próximo a los 30 billones de toneladas, que repartidas de manera uniforme serían 50 kilogramos por metro cuadrado de la superficie terrestre ${ }^{19}$.

Según quienes pretenden evadir el enfoque político de la crisis antropocénica, ella ha surgido globalmente sin vínculo con las particularidades de regímenes socioeconómicos, geográficas e histórico-culturales del mundo; sin embargo, deberían detenerse en explicar por qué la llamada tecnosfera tiende a crecer y pesar insosteniblemente en el sistema Tierra ${ }^{20}$, al traer consigo, a la vez que desarrollo tecnológico y nuevos productos de consumo humano, agotamiento o deterioro de recursos naturales, contaminación ambiental, desigualdades socioeconómicas y conflictos bélicos. Se percatarían entonces de que es imposible pasar por alto las causas histórico-sociales y políticas de los problemas que caracterizan al Antropoceno, cuyo comienzo coincide, precisamente, con el auge del capitalismo en Europa Occidental y su posterior expansión hacia otras regiones del mundo.

\footnotetext{
18 Plitt, «¿Qué es el Antropoceno...»

19 «¿Qué es la tecnosfera?», Alberto Cajal, acceso: 23 de setiembre de 2020, https://www.lifeder.com/ tecnosfera/

20 «El peso insostenible de la tecnosfera», Jan Zalasiewicz, Correo de la Unesco, 2018-2, acceso: 20 de setiembre de 2021, https://es.unesco.org/courier/2018-2/peso-insostenible-tecnosfera
} 
El autor de estas reflexiones concuerda con Malm ${ }^{21}$ cuando afirma que el mundo capitalista actual es un segmento ínfimo de la especie humana, el cual posee los medios de producción y pretende tomar las decisiones trascendentales sobre la utilización de los recursos y las tecnologías. Esa minoría solo tiene un objetivo: enriquecerse aún más. Desde un punto de vista capitalista, lo que se debe hacer (en el Antropoceno) es invertir en la producción y consumo de todo lo que genere ganancias económicas. En este modo de pensar y proceder se encuentra el origen del calentamiento global y de otros impactos nefastos de la humanidad sobre el medio ambiente.

Resulta interesante la coincidencia de ideas entre Engels, filósofo del siglo XIX, y Chakrabarty, historiador del siglo XXI, en cuanto a las bases de partida para buscar soluciones a la crisis del Antropoceno. Pensaba Engels ${ }^{22}$ que: «para llegar a esta regulación (n. a. de la actividad humana sobre la naturaleza y la sociedad misma), no basta con el mero conocimiento. Hace falta, además, transformar totalmente al régimen de producción vigente hasta ahora y, con él, todo nuestro orden social presente». Por su parte, afirma Chakrabarty, actualmente, que ${ }^{23}$ : «No podemos mantener la forma actual del capitalismo durante 100 o 200 años más (...) Tenemos que asumir la responsabilidad de transmitir este mensaje en las escuelas y universidades».

La gravedad de los impactos humanos sobre el funcionamiento y equilibrio de la Tierra ha convocado a la humanidad hacia la búsqueda de soluciones. Desde la década de 1970, las máximas autoridades de los países del mundo son convocadas por la Organización de Naciones Unidas (ONU) para establecer políticas y estrategias que pongan freno a la creciente crisis ambiental, en medio de la cual

el medio ambiente planetario está reduciendo su capacidad de reversibilidad, de eliminar las perturbaciones ambientales, porque está perdiendo la propiedad de autorregulación; el medio ambiente natural no puede soportar más los impactos y acciones humanas y surgen los problemas ambientales naturales y socioculturales. $^{24}$

Uno de los mensajes más trascendentes y recordados de los líderes políticos que han intervenido en estas reuniones mundiales sobre medio ambiente y desarrollo, es el de Fidel Castro Ruz ${ }^{25}$ en la Cumbre de la Tierra o Cumbre de Río, celebrada en Río de Janeiro el 2 de junio de 1992; allí expresó:

Una importante especie biológica está en riesgo de desaparecer por la rápida y progresiva liquidación de sus condiciones naturales de vida (...) Los bosques

\footnotetext{
21 «Desastre en Dominica: ¿El Antropoceno o el Capitaloceno?», Andreas Malm, Correo de la Unesco, 2018-2, acceso: 20 de setiembre de 2021, https://es.unesco.org/courier/2018-2/desastre-dominicaantropoceno-o-capitaloceno

22 Engels, Dialéctica de la naturaleza..., 154.

23 Sidhva, «La humanidad es...»

24 N. Sosa, «Perspectiva ética», Colección Monografías de Educación Ambiental, Fundación UniversidadEmpresa (Madrid: UNED, 1995), 67.

25 Fidel Castro, Discurso pronunciado ante la Conferencia de la ONU sobre Medio Ambiente y Desarrollo (La Habana: Editora Política, 1992), 1-3.
} 
desaparecen, los desiertos se extienden, miles de millones de toneladas de tierra fértil van a parar cada año al mar. Numerosas especies se extinguen (...) Menos lujo y menos despilfarro en unos pocos países para que haya menos pobreza y menos hambre en gran parte de la Tierra (...) Hágase más racional la vida humana (...) Utilícese toda la ciencia necesaria para el desarrollo sostenido sin contaminación. Páguese la deuda ecológica y no la deuda externa. Desaparezca el hambre y no el hombre.

En esta importante reunión se aprobaron documentos fundamentales como la Agenda 21 para el Desarrollo Sostenible, mediante la cual se pretende que los países dependientes logren salir del subdesarrollo y satisfagan necesidades básicas de la población, lo cual requiere la movilización económica de los recursos y servicios ambientales (naturales y humanos), aplicar modernas tecnologías, construir capital económico, sociocultural y recuperar o mejorar el capital natural; pero dichos procesos enfrentan obstáculos de todo tipo. La dependencia externa y la insolvencia económica de esos países los hace aceptar estilos «extractivistas» en la explotación de los recursos naturales que expolian por completo, con vistas a amortizar sus necesidades, pero que en fin de cuentas se dirigen a proveer de ganancias a los poderes geopolíticos y geoeconómicos que dominan los territorios ${ }^{26}$.

Lamentablemente, a casi 40 años después de la Cumbre de Río, la situación ambiental del mundo no muestra mejoras sustanciales. En 2015, el Papa Francisco, en su encíclica Laudato Si o Alabado seas, realizó un análisis del modelo actual de desarrollo, la cultura del descarte y del mito del progreso. Criticó con agudeza las visiones tecnocráticas, la economía verde, la hegemonía del capital financiero y el consumismo e individualismo; propuso una visión ecológica integral de la vida y una sensibilización subjetiva respecto de la naturaleza, coincidente en que, por su esencia, el capitalismo es el causante de la crisis ambiental, aunque no lo declare de manera directa ${ }^{27}$.

La emergencia de la crisis ambiental contemporánea (argumento principal de la llegada al Antropoceno) indica, según J. Mateo ${ }^{28}$, que:

Es necesario perfilar un nuevo tipo de sociedad y un nuevo modelo y estilo de desarrollo que sustituya al capitalismo. Se hace necesario formular, en contraposición al paradigma capitalista, un paradigma ambientalista post capitalista, en que se priorice la democracia participativa, la ecología socialista, el anti productivismo y la diversidad cultural.

El académico antes citado argumenta que Cuba es un ejemplo único de país en vías de desarrollo, a nivel mundial, donde desde hace más de 60 años se ha

\footnotetext{
26 Aramis Latichinian, El ambientalista crítico. Ante el nuevo escenario de América Latina (Lima: Universidad Inca Garcilaso de la Vega, 2014), 161.

27 L. Martínez, «A propósito de la encíclica "Laudato Si" del Papa Francisco», http//www. rebelión.org, 07-122015, 5.

28 José Mateo, Cuba: los retos de un país en Revolución para enfrentar la crisis ambiental (La Habana: Editorial Nuevo Milenio, 2015), 37.
} 
tratado de construir un proceso de desarrollo dirigido no solo a solventar la crisis ambiental, sino a lograr, sobre la base de la justicia social, la equidad y la solidaridad, garantizar las necesidades básicas de su población, en un modelo de desarrollo en el cual la revolución, desde su comienzo, implicó un cambio radical de las estructuras de poder, enfrentando vicisitudes de todo tipo, geopolíticas, económicas, socioculturales y ambientales.

Los logros cubanos en cuanto a la gestión ambiental para el desarrollo sostenible son reconocidos por la ONU y entre ellos merecen mención los siguientes 29 :

-Se eliminó el analfabetismo.

-Se erradicó la pobreza extrema.

-Se incrementó la superficie boscosa del país.

-Se produjo una transformación total de la estructura agraria.

-Se instrumentaron programas contra enfermedades infecciosas.

-Disminuyó la mortalidad infantil y se incrementó la esperanza de vida.

-Se mejoraron substancialmente las condiciones ambientales básicas, incrementándose la cobertura de población con agua potable, saneamiento, servicios de electricidad, entre otros.

-Se desarrolló un proceso de ordenamiento territorial que permitió realizar evaluaciones donde se valoraba el componente ambiental en la mayoría de las inversiones de carácter socioeconómico.

-Se ha logrado, gracias a un alto desarrollo científico, la evaluación del potencial de los recursos naturales del país, el desarrollo de vacunas y nuevos medicamentos, tecnologías para el aprovechamiento de residuales, incluidas las prácticas de producciones limpias, la rehabilitación y restauración de ecosistemas afectados, la mejor gestión en el manejo de los recursos como agua y suelo, entre otros.

-Además, Cuba está involucrada, de forma activa, en todo el proceso de integración global que demanda el enfrentamiento efectivo a la crisis ambiental, mediante su participación en todos los convenios y acuerdos internacionales existentes, cuyos compromisos procura implementar con agilidad mediante la Estrategia Ambiental Nacional y otros instrumentos de la gestión ambiental para el desarrollo sostenible.

\footnotetext{
29 Ministerio de Ciencia, Tecnología y Medio Ambiente de Cuba (Citma), «Estrategia Ambiental Nacional
} (2016-2020)», La Habana, 2015, 34. 
La experiencia cubana es útil para el mundo porque muestra que, no obstante todos los esfuerzos que para desarrollar un país por la senda de la sostenibilidad, se enfrenta con la necesidad de transformar, manejar y utilizar el medio ambiente desde una mirada diferente a la del capitalismo neoliberal, donde la economía de mercado intensifica y hace incontrolable la actividad humana sobre la naturaleza y la sociedad misma, en su búsqueda interminable de ganancias, convirtiendo todo en mercancía. También evidencia que, a pesar de la gravedad de la crisis ambiental contemporánea, la humanidad, con su desarrollo científico-técnico y eficiente gobernabilidad, puede tomar medidas para controlar, adaptarse, mitigar o prevenir los impactos humanos sobre el medio ambiente, entre los que se destaca el calentamiento global y el cambio climático en general.

\section{Una nueva forma de pensar y actuar}

Frente a la grave problemática ambiental, interpretada por muchos como prueba de que el homo sapiens se ha convertido en una fuerza geológica planetaria capaz de provocar daños y desequilibrios muy poco favorables para su futuro en la Tierra, se impone que la humanidad replantee formas de pensar y de actuar con respecto a cómo interactuar y utilizar el medio ambiente en el que vive y del cual vive. Urge construir una nueva cultura ambiental, sustentada en una visión ecocéntrica y humanística del medio ambiente, basada en el manejo racional y la relación armónica (ecosistémica) entre naturaleza y sociedad humana, global y localmente. En este sentido, el autor, impulsado por su vocación pedagógica y sus experiencias investigativas, pretende resaltar, en dicha construcción, el papel de la educación de cada persona, en interrelación con las dimensiones políticas, científico-tecnológicas, legales, éticas o estéticas de este complejo proceso.

El estudio crítico realizado por este autor sobre el estado actual de la cultura ambiental para el desarrollo sostenible en profesores y estudiantes de carreras pedagógicas en la Universidad de Matanzas, reveló que su mejora demanda prestar atención a la débil implicación personal de estos, en conocer y solucionar problemas ambientales de sus contextos de vida. Ante la situación antes planteada, se elaboró un sistema de indicadores que facilita la autovaloración del desarrollo de la cultura ambiental para el desarrollo sostenible, como una de las vías para fomentar el protagonismo, la implicación personal y la responsabilidad ciudadana y profesional en la construcción de sus saberes, fundamentos éticos y comportamientos medioambientales.

Dichos indicadores fueron derivados de una meditada definición de la variable cultura ambiental para el desarrollo sostenible, labor realizada por un grupo de investigadores en el proyecto científico: cultura ambiental con enfoque sostenible y preparación profesional pedagógica para educarla desde la escuela, los cuales deberán ser debatidos y reformulados en un taller antes de proceder a la autovaloración reflexiva y crítica del estado actual de la cultura ambiental de cada participante. Se evidenció que este procedimiento contribuye a la formación ambiental, ya que permite cumplir diversas funciones didácticas y 
educativas. Se ha implementado en la formación inicial de docentes, en postgrado, así como en la extensión cultural, con gran aceptación de estudiantes y docentes.

La variable de cultura ambiental para el desarrollo sostenible fue definida como 30 :

El conjunto de rasgos distintivos, espirituales y materiales, intelectuales, afectivos y comportamentales que caracterizan los modos de interacción hombre-sociedad-naturaleza en una sociedad, un grupo social o en una persona y que son determinantes en la magnitud y el signo de su impacto sobre el medio ambiente donde se desarrolla.

La cultura ambiental para el desarrollo sostenible contempla tres dimensiones dialécticamente entretejidas: saberes ambientales indispensables, una ética ambiental de justicia y responsabilidad, así como capacidades de gestión ambiental generales y profesionales; todas estas son correspondientes con las esferas cognitiva, afectiva y conductual del desarrollo personal.

Conviene que cada ciudadano aprenda a autoevaluar el nivel de desarrollo de su cultura ambiental enfocada al desarrollo sostenible $\mathrm{y}$, a partir de su auto percepción, se disponga a mejorarla, para lo cual se sugiere el siguiente sistema de indicadores ${ }^{31}$ que permiten caracterizar, de modo personal, las dimensiones antes apuntadas ${ }^{32}$ :

Dimensiones e indicadores para la autoevaluación de la cultura ambiental

En la dimensión

cognoscitiva o de los

saberes ambientales

generales se proponen los

siguientes indicadores:

-Conoce qué es el medio ambiente: su importancia, enfoques para su gestión y los componentes que se le reconocen en la actualidad.

\section{En la dimensión ética-} ambiental, cuyos indicadores se refieren a sí:

Muestra actitudes consecuentes con los esfuerzos por alcanzar el equilibrio medio ambientedesarrollo sostenible: indagación, actualización, difusión, comunicación, valoración, implicación y cooperación.
En la dimensión

comportamental o

conductual, referida a la

aplicación en la práctica, los indicadores propuestos son:

-Manifestación de comportamientos cotidianos responsables, que evitan la aparición o agravamiento de problemas medioambientales.

30 Wilfredo Mesa, «Cultura ambiental con enfoque sostenible y preparación profesional pedagógica para educarla desde la escuela», Informe final de proyecto de investigación, Universidad de Matanzas. En soporte digital, 2015: 28.

31 Wilfredo Mesa y Rosa M. Fajardo, «Taller para autoevaluar la cultura ambiental». En memorias de Taller Internacional de Educación Ambiental Educambie 2017, Universidad de Matanzas, 2017.

32 Teresita Maldonado, Modelo de evaluación y acreditación de los Centros de Educación y Cultura ambiental (Ciudad de México: Secretaría de Medio Ambiente y Recursos Naturales, 2009). 
En la dimensión

cognoscitiva o de los

saberes ambientales

generales se proponen los

siguientes indicadores:

-Identifica los problemas medioambientales a diferentes

escalas: global, regional, nacional, local, sus causas y consecuencias, así como medidas de prevención, solución o mitigación.

-Razona sobre el desarrollo sostenible como salida a la crisis ambiental: sus fundamentos generales (filosóficos, sociológicos, psicológicos, tecnológicos) para estudiar la relación medio ambiente-desarrollo desde la perspectiva de la sostenibilidad.

-Establece una adecuada relación entre cultura ambiental, educación ambiental y escuela, así como una adecuada valoración del papel mediador de los educadores en su formación.
En la dimensión éticaambiental, cuyos indicadores se refieren a sí:

-Defiende valores morales que orientan el comportamiento personal y profesional hacia el desarrollo sostenible: laboriosidad, responsabilidad, profesionalidad, identidad y solidaridad, entre otros.
En la dimensión

comportamental o

conductual, referida a la

aplicación en la práctica, los

indicadores propuestos son:

-Participación activa en la búsqueda de solución o mitigación a problemas medioambientales locales y de su entorno más cercano.

\section{-Expresa sentimientos que} evidencian apego a las ideas del desarrollo sostenible: amor a la naturaleza y a la obra creada por el ser humano, rechazo a los actos que agreden el medio ambiente, deseos de estar informado y de participar en la solución de problemas medioambientales.

-Posee alto sentido del deber de incorporar sistémicamente la dimensión ambiental en los procesos de su cotidiano de vida y actividad profesional.

\section{-Noción de las características} del estado ambiental de su entorno más cercano, así como de la cultura ambiental que prevalece en sus habitantes.
-Reconoce a la educación ambiental como dimensión de la educación integral de la personalidad de cada individuo.
-Incorporación efectiva de la dimensión ambiental (planificación, ejecución y control) en todos los procesos de su vida cotidiana y profesional.

Fuente: Elaboración propia.

Después de comprender el contenido y alcance de los indicadores propuestos, ¿Cómo debe proceder la persona para aproximarse al autoconocimiento del estado de su cultura ambiental? Pues, este deberá ser un acto de honestidad individual, reflexión autocrítica y autoconocimiento, proyectado hacia el mejoramiento de la persona ${ }^{33}$ en el plano de su relación con el medio ambiente en que vive. Las categorías por utilizar serían de corte cualitativo, cada indicador podrá ser valorado de: muy favorable, favorable o poco favorable y, finalmente, se hace un balance aproximativo, tomando en cuenta las categorías predominantes; aunque lo más importante es que ocurra la mirada crítica hacia

\footnotetext{
33 Norma Cárdenas, «La formación de alumnos activos y reflexivos en el proceso de enseñanza-
} aprendizaje» (Congreso Internacional Pedagogía, La Habana, Cuba, 3 al 7 de febrero de 2003). 
uno mismo, se tracen metas para mejorar la cultura ambiental y se estimulen los deseos de impactar positivamente sobre el medio ambiente.

De vuelta al debate científico sobre el Antropoceno, el autor de estos apuntes reconoce que es importante porque los científicos intentan configurar un modelo global que explique el funcionamiento actual del sistema terrestre ${ }^{34}$, altamente impactado por la tecnosfera; pero, es más importante trabajar para que las nuevas generaciones adquieran una nueva cultura ambiental y se dispongan resueltamente a vencer los grandes desafíos del futuro, para que en una fecha no tan lejana, se vuelva al debate sobre qué nombre otorgar a la nueva etapa geológica caracterizada porque el humano pudo convertirse en una fuerza geológica a favor de la relación armónica entre el desarrollo de la sociedades humanas y la naturaleza.

\section{Conclusiones}

La gran magnitud e intensidad alcanzada por la actividad humana sobre la Tierra es innegable; no obstante, son insuficientes las evidencias geológicas de sus impactos duraderos, caracterizados por una desigual distribución espacial y temporal. Por tal motivo, el Antropoceno no está legitimado como concepto geocronológico, aunque es de gran utilidad porque suscita polémicas que incentivan la reflexión global sobre la impostergable unidad de los esfuerzos políticos, científicos, tecnológicos y sociales que la humanidad necesita para reorientarse hacia la armonía con la naturaleza y superar su actual crisis civilizatoria.

Lo más relevante de intervenir en el debate acerca del Antropoceno es que indica un camino para superar la crisis civilizatoria actual, cuyos problemas más preocupantes son los medioambientales: la necesidad urgente de construir una nueva cultura ambiental que facilite pasar de una visión antropocéntrica y productivista, basada en la falsa idea del dominio sobre la naturaleza, a una ecocéntrica y humanística, basada en la armonía y manejo racional de los servicios ambientales y de la sociedad humana. Ese cambio cultural involucra a todos los países, todos los sectores de la sociedad y a cada persona.

Las nuevas generaciones tienen grandes retos que vencer en el futuro antropocénico y los humanos de hoy deberán sembrar los saberes, las actitudes, los sentimientos y las voluntades (aspectos no dados al homo sapiens por herencia biológica, sino sociocultural) que le permitan lograrlo mediante políticas integrales a escala global, en las que el ser humano sea la prioridad y no las ganancias económicas o las ventajas políticas.

34 Issberner y Léna, «Antropoceno: la problemática...» 


\section{Formato de citación según APA}

Mesa-Ortega, W. R. (2021). Antropoceno, ¿Última unidad geocronológica o Ilamamiento a la nueva cultura ambiental que necesita la humanidad? Revista Espiga, 20 (42), páginas 99-118.

\section{Formato de citación según Chicago-Deusto}

Mesa-Ortega, Wilfredo Ricardo. «Antropoceno, ¿Última unidad geocronológica o Ilamamiento a la nueva cultura ambiental que necesita la humanidad?». Revista Espiga 20, n.o 42 (julio-diciembre, 2021): páginas 99-118. 


\section{Referencias}

Cárdenas, Norma. «La formación de alumnos activos y reflexivos en el proceso de enseñanza-aprendizaje». Congreso Internacional Pedagogía, La Habana, Cuba, 3 al 7 de febrero de 2003.

Castro, Fidel. Discurso pronunciado ante la Conferencia de la ONU sobre Medio Ambiente y Desarrollo. Editora Política. La Habana, 1992, 1-3.

Chaparro, Jeffer e Ignacio Meneses. «El Antropoceno: aportes para la comprensión del cambio global». Ar@cne. Revista electrónica de recursos en internet sobre Geografía y Ciencias Sociales, n. 203 (2015).

Engels, Federico. Dialéctica de la naturaleza. El papel del trabajo en el proceso de transformación del mono en hombre. Editora Política. La Habana. 1979.

Cajal, Alberto. «¿Qué es la tecnosfera?». Acceso: 23 de setiembre de 2020. https://www.lifeder.com/tecnosfera/

Issberner, Liz-Rejane y Philippe Léna. «Antropoceno: la problemática vital de un debate científico». Correo de la Unesco, 2018-2. Acceso: 20 de setiembre de 2021. https://es.unesco.org/courier/2018-2/antropoceno-problematicavital-debate-cientifico

Latichinian, Aramis. El ambientalista crítico. Ante el nuevo escenario de América Latina. Lima: Universidad Inca Garcilaso de la Vega, 2014.

Maldonado, Teresita. Modelo de evaluación y acreditación de los Centros de Educación y Cultura ambiental. Ciudad de México: Secretaría de Medio Ambiente y Recursos Naturales, 2009.

Malm, Andreas. «Desastre en Dominica: ¿El Antropoceno o el Capitaloceno?». Correo de la Unesco, 2018-2. Acceso: 20 de setiembre de 2021. https:// es.unesco.org/courier/2018-2/desastre-dominica-antropoceno-ocapitaloceno

Martí, José. Obras Completas: Discurso en el Liceo Cubano, Tampa. Tomo 4. La Habana: Editorial de Ciencias Sociales, 1991.

Martí, José. Obras Completas: Maestros ambulantes. Tomo 8. La Habana: Editorial de Ciencias Sociales, 1991.

Martínez, L. A propósito de la encíclica Laudato Si del Papa Francisco. http//www. rebelión.org, 07-12-2015, 5.

Mateo, José. Cuba: los retos de un país en Revolución para enfrentar la crisis ambiental. La Habana: Editorial Nuevo Milenio, 2015. 
Mateo, José. Medio Ambiente y Desarrollo. Universidad de La Habana. Facultad de Geografía. La Habana. En soporte digital, 2002.

Mesa, Wilfredo y Rosa María Fajardo. Taller para autoevaluar la cultura ambiental. En memorias de Taller Internacional de Educación Ambiental Educambie 2017. Universidad de Matanzas, 2017.

Mesa, Wilfredo. Cultura ambiental con enfoque sostenible y preparación profesional pedagógica para educarla desde la escuela. Informe final de Proyecto de investigación. Universidad de Matanzas. En soporte digital, 2015.

Mesa, Wilfredo. Geología Elemental. Material de consulta de curso optativo. Licenciatura en Educación Biología-Geografía y Biología-Química. Universidad de Matanzas. En soporte digital, 2017.

Ministerio de Ciencia, Tecnología y Medio Ambiente de Cuba (Citma). Estrategia Nacional Ambiental (2016-2020). La Habana, 2015, 34.

Plitt, Laura. "¿Qué es el Antropoceno, la "edad de los humanos" que expertos aseguran hemos entrado?». Acceso: 18 de agosto de 2020. https:// www.bbc.com/mundo/noticias-37220892

Sidhva, Shiraz. «La humanidad es una fuerza geológica». Correo de la Unesco, 2018-2. Acceso: 20 de setiembre de 2021. https://es.unesco.org/courier/ 2018-2/humanidad-es-fuerza-geologica

Sosa, N. «Perspectiva ética». Colección Monografías de Educación Ambiental, Fundación Universidad-Empresa. Madrid: UNED, 1995, 67.

Unesco. «Léxico del Antropoceno». Acceso: 20 de setiembre de 2021. https:// es.unesco.org/courier/2018-2/lexico-del-antropoceno

Zalasiewicz, Jan. «El peso insostenible de la tecnosfera». Correo de la Unesco, 2018-2. Acceso: 20 de setiembre de 2021. https://es.unesco.org/courier/ 2018-2/peso-insostenible-tecnosfera 